Document downloaded from:

http://hdl.handle.net/10251/64208

This paper must be cited as:

Correia, DM.; Ribeiro, C.; Ferreira, JCC.; Botelho, G.; Gómez Ribelles, JL.; Lanceros Mendez, S.; Sencadas, V. (2014). Influence of electrospinning parameters on Poly(hydroxybutyrate) electrospun membranes fiber size and distribution. Polymer Engineering and Science. 54(7):1608-1617. doi:10.1002/pen.23704

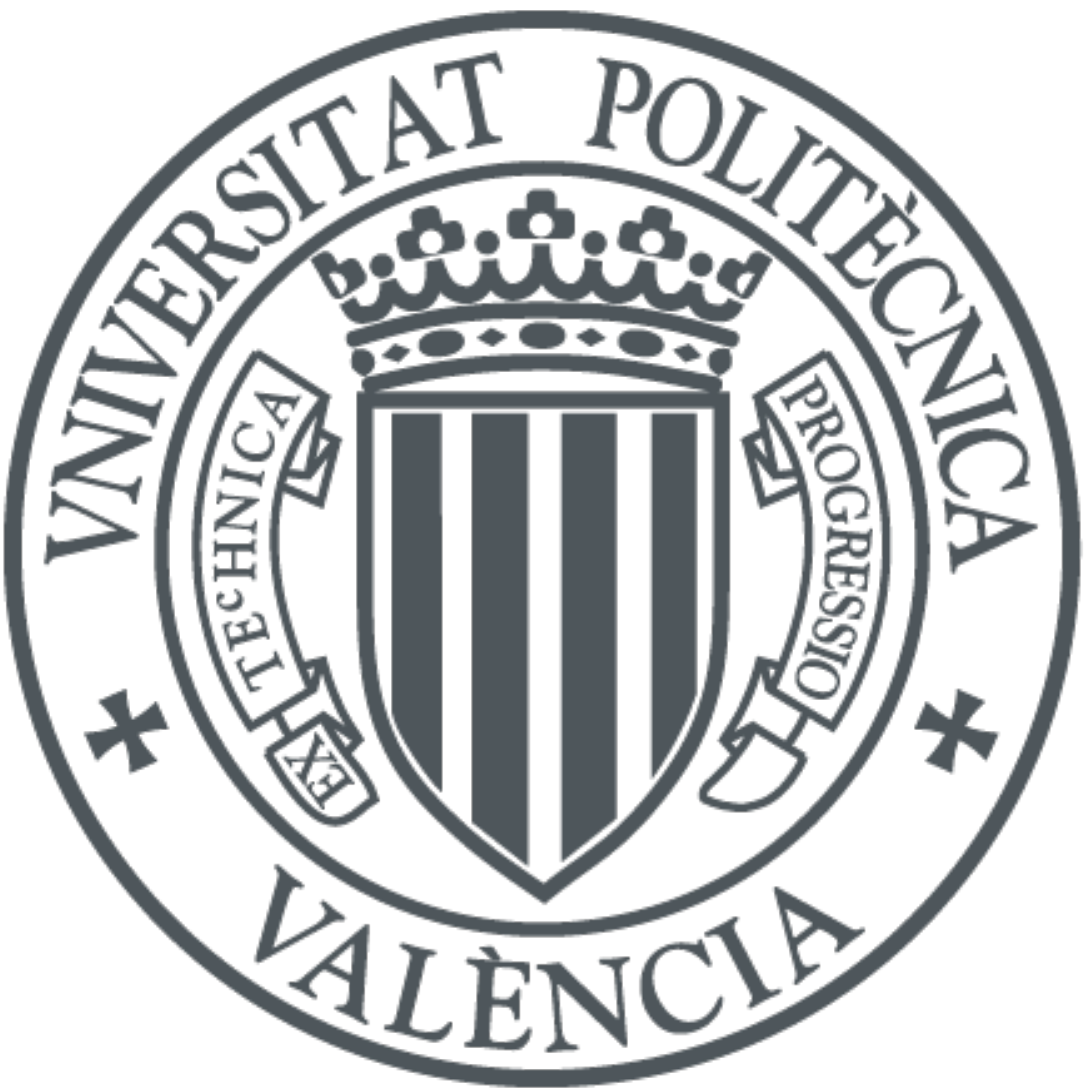

The final publication is available at

http://dx.doi.org/10.1002/pen.23704

Copyright Wiley

Additional Information 


\title{
Influence of electrospinning parameters on Poly(hydroxybutyrate) electrospun membranes fiber size and distribution
}

D. M. Correia ${ }^{1,2}$, C. Ribeiro ${ }^{1}$, J. C. Coelho ${ }^{1}$, G. Botelho ${ }^{2}$, J. L. Gomez Ribelles ${ }^{3,4}$, S. Lanceros-Méndez ${ }^{1,5}$ and V. Sencadas ${ }^{1,6 ; *}$

${ }^{1}$ Centro/Departamento de Física da Universidade do Minho, Campus de Gualtar, 4710057 Braga, Portugal.

2 Dept. Química, Centro de Química, Universidade do Minho, Campus de Gualtar, 4710-057 Braga, Portugal;

${ }^{3}$ Center for Biomaterials and Tissue Engineering, Universitat Politècnica de València, Camino de Vera s/n, 46022 Valencia, Spain

${ }^{4}$ Ciber en Bioingeniería, Biomateriales y Nanomedicina (CIBER-BBN), Valencia, Spain

5 INL - International Iberian Nanotechnology Laboratory, 4715-330 Braga, Portugal

${ }^{6}$ Escola Superior de Tecnologia, Instituto Politécnico do Cávado e do Ave, Campus do IPCA, 4750-810, Barcelos, Portugal.

e-mail: vsencadas@fisica.uminho.pt

\begin{abstract}
Poly(hydroxybutyrate) (PHB) obtained from sugar cane was dissolved in a blend of chloroform and dimethylformamide (DMF) and electrospun at $40^{\circ} \mathrm{C}$. By adding DMF to the solution, the electrospinning process for the PHB polymer becomes more stable, allowing complete polymer crystallization during the jet travelling between the tip and the grounded collector.

The influence of processing parameters on fiber size and distribution was systematically studied. It was observed that an increase of tip inner diameter promotes a decrease of the fiber average size and a broader distribution. On the other hand, an increase of the
\end{abstract}


electric field and flow rate produces an increase of fiber diameter until a maximum of $\sim 2.0 \mu \mathrm{m}$, but for electric fields higher than $1.5 \mathrm{kV} . \mathrm{cm}^{-1}$, a decrease of the fiber diameter was observed. Polymer crystalline phase seems to be independent of the processing conditions and a crystallinity degree of $53 \%$ was found. Moreover, thermal degradation of the as-spun membrane occurs in single step degradation with activation energy of 91 $\mathrm{kJ} / \mathrm{mol}$. Furthermore, MC-3T3-E1 cell adhesion was not inhibited by the fiber mats preparation, indicating their potential use for biomedical applications.

\section{Keywords}

\section{Introduction}

Tissue engineering is a multidisciplinary field based on the knowledge of physics, chemistry, material science and engineering, biology and medicine for the reconstruction, maintenance or improving tissue/organ functions [1]. Materials used in tissue engineering should possess properties that assure a suitable physical support and substrate for growth factors, cell adhesion, proliferation and differentiation of the primary cells during in vitro and in vivo assays [2].

In recent years, much attention has been paid to the production, processing and applications of polyhydroxyalkanoates (PHAs) in the biomedical field. PHAs are natural polymers synthesized by a wide variety of microorganisms such as soil bacteria, blue-green algae and genetically modified plants, being the poly(hydroxybutyrate) (PHB) the most common type of PHAs [3].

PHB was discovered by Lemoigne in the bacterium Bacillus megaterium in 1926 [4, 5]. It was the first polymer of the PHAs class to be discovered and it is also the most widely studied and the best characterized PHA. PHB is a non-toxic, insoluble in water, biodegradable and biocompatible thermoplastic polymer with a high crystallinity degree and with physical properties similar to polypropylene [5-7]. The high crystallinity of PHB (almost $80 \%$ ) is a consequence of its perfect stereoregularity and high purity, resulting from its bacterial fermentation production [8].

Due to its natural origin and its properties, PHB is suitable for biomedical applications. It has been already evaluated for controlled drug release systems, surgical structures, wound dressings, orthopedic devices, tissue engineering and skin substitute materials, 
among others [9]. However, the high crystallinity and brittleness of PHB limits its application potential [5]. The high nucleation rate and smaller number of PHB nuclei often results, during crystallization, large spherulites that exhibit inter-spherulitic cracks. Polymer glass transition occurs near $0{ }^{\circ} \mathrm{C}$ which is below room temperature, and secondary crystallization of the amorphous phase occurs during storage [8].

For tissue engineering applications, studies with PHB have shown that cells such as osteoblasts, epithelial cells and ovine chondrocytes adhered efficiently to PHB films synthesized by both solvent casting and solute-leaching techniques [3]. Furthermore, composites of amorphous carbonated apatite (ACP) and poly-(R)-3-hydroxybutyrate contribute to cell proliferation [8] and PHB with hydroxyapatite (HAP) composites improves osteoblasts cell growth [10].

Due to the promising application of PHB in tissue engineering applications, further studies have been conducted in electrospun PHB fibers, as electrospun polymeric membranes have attracted an increasing interest for tissue engineering applications due to their nanostructured morphology, which shall mimic the random distribution of the fibrillar extracellular matrix components like collagen [9] or elastin [11]. Furthermore, electrospun membranes have large specific surface area and high porosity, which are desirable properties for nutrient delivery, fluid absorption excretion, and oxygen supply [9].

Ultrafine fibers of PHB, PHBV (poly(hydroxybutyrate-co-hydroxyvalerate)) and their blends have been produced using chloroform as a solvent system and its viability was evaluated with mouse fibroblasts [12], and used for bone scaffolds, especially cell attachment, proliferation and alkaline phosphatase (ALP) activity of human osteoblasts [3].

Fiber mats of PHB have been prepared under different conditions, including spinning PHB solution with chloroform as a solvent [13], producing three-dimensional nanofibers nonwoven webs from solution of PHB and dodecylbenzene sulfonic acid (DBSA) doped polyaniline in chloroform/trifluoroethanol mixture [14] and electrospinning pure polyvinyl alcohol (PVA), PHB and their blends using a solvent system of 1, 1, 1, 3, 3, 3-hexafluoro-2-propanol (HFIP) [9].

Despite the aforementioned investigations and the large application potential of these materials for tissue and biomedical engineering, there is a lack of systematic study on how processing parameters modify membrane fiber dimensions which is one of the key 
features for tailoring membranes for specific applications. In the present work PHB mats were processed by electrospinning and a systematic study of the influence of electrospinning parameters such as applied voltage, needle inner diameter and solution flow rate on fiber morphology is presented. The suitability of the developed membranes for biomedical applications was proven by cell viability studies performed with osteoblast-like MC3T3-E1 cells.

\section{Experimental}

Materials: Poly(hydroxybutyrate), (PHB, molecular weight of $\sim 531112 \mathrm{Da}$ ) from sugar cane was supplied by PHB Industrial and dissolved in a blend of $\mathrm{N}, \mathrm{N}$ dimethylformamide (DMF, from Merck) and Chloroform (CF, from Merck) (3/7 v/v), at $60{ }^{\circ} \mathrm{C}$ under stirring until complete polymer dissolution, to achieve a polymer concentration of 7 and $10 \mathrm{wt} \%$ of the final solution.

Electrospinning: The polymer solution was placed in a commercial plastic syringe (10 $\mathrm{mL}$ ) fitted with a steel needle with different diameters (from $0.5 \mathrm{~mm}$ up to $1.7 \mathrm{~mm}$ ). Electrospinning was conducted at $40{ }^{\circ} \mathrm{C}$ at different electrical fields created by a high voltage power supply from Glassman (model PS/FC30P04). A syringe pump (from Syringepump) was used to feed the polymer solutions into the needle tip at a rate between 5 and $20 \mathrm{~mL} \cdot \mathrm{h}^{-1}$. The as-spun random oriented fibers were collected in grounded collecting plate.

A PHB film was produced by solvent casting from the same solution at $60{ }^{\circ} \mathrm{C}$, because films crystallized during solvent evaporation at temperatures below that presented high porosity and were unstable due to their poor mechanical properties.

Characterization: Electrospun fibers were coated with a thin gold layer using a sputter coating (Polaron, model SC502) and their morphology was analyzed using scanning electron microscopy (SEM) (Cambridge, Leica) with an accelerating voltage of $15 \mathrm{kV}$. The fibers average diameter and their size distribution was calculated over approximately 40 fibers using de SEM image (2000X magnification) and the Image J software. 
Infrared measurements (FTIR) were performed at room temperature in an ABB FTLA 2000 apparatus in transmission mode from 4000 to $500 \mathrm{~cm}^{-1}$. FTIR spectra were collected after 32 scans with a resolution of $4 \mathrm{~cm}^{-1}$. Contact angle measurements (sessile drop in dynamic mode) were performed at room temperature in a Data Physics OCA20 device using ultrapure water as test liquid. The contact angles were measured by depositing water drops $(3 \mu \mathrm{L})$ on the sample surface and analyzed with SCA20 software. At least 6 measurements in each PHB sample were performed in different sample locations and the average contact angle was taken as the result for each sample. The thermal degradation kinetics of PHB was characterized by means of thermogravimetric analysis in a Perkin-Elmer Pyris-1 TGA apparatus using different heating rate scans. All experiments were performed under a nitrogen atmosphere. Differential scanning calorimetry measurements (DSC) were performed in a PerkinElmer Pyris-1 apparatus at a heating rate of $10{ }^{\circ} \mathrm{C} \cdot \mathrm{min}^{-1}$. The samples for the DSC studies were cut into small pieces from the middle region of the electrospun membranes and placed into $40 \mu \mathrm{L}$ aluminum pans. All experiments were performed under a nitrogen purge.

Cell culture: For cell culture, circular PHB nanofiber membranes with $13 \mathrm{~mm}$ of diameter were prepared. For sterilization purposes, the nanofibers were immersed in 70 $\%$ ethanol for $30 \mathrm{~min}$ several times. Then, the membranes were washed 5 times for 5 min with phosphate-buffered saline solution (PBS) followed by washing 3 times with the culture medium to eliminate any residual ethanol.

MC3T3-E1 cells (Riken cell bank, Japan) were cultivated in Dulbecco's modified Eagle's medium (DMEM) 1g/L glucose (Gibco) containing 10 \% Fetal Bovine Serum (FBS) (Fisher) and $1 \%$ penicillin/streptomycin (P/S).

For the study of cell viability, the osteoblast-like cells were seeded in 24 -well TC plates with PHB fiber membranes at cell density of $3 \times 10^{4}$ cells/well for 2 days. For the quantification of cell viability, MTT assay (Sigma-Aldrich) was carried out.

\section{Results and Discussion}

a) Fiber mat morphology and average fiber diameter 
The parameters having influence on the morphology and properties of the electrospun fibers can be divided in three main groups: initial polymer solution, jet formation and collection procedure [15]. Among the parameters related to the polymer solution, the most relevant are the nature of used solvent (dielectric properties, volatility, boiling point and others), the solution concentration, that controls its viscosity, and the molecular weight of the polymer (that must allow polymer entanglement). Moreover, parameters that control the jet formation, stability and solvent evaporation are the flow rate through the needle, needle inner diameter, distance from the needle to collector, temperature, moisture and applied voltage. With respect to the collection procedure, it can be static or rotating collector, and in the last case the rotating speed of the collector is the most important parameter determining fiber orientation and diameter [15-17]. The number of parameters that influence the electrospinning process is quite high and in order to allow a broad characterization of the influence of the electrospinning parameters on fiber size and distribution some of them should be fixed.

In order to obtain well-formed fibers and samples without bead defects, a blend of $\mathrm{CF} / \mathrm{DMF}$ solvents was used to dissolve the PHB polymer. CF was used to dissolve the polymer, but it has low dielectric constant and boiling point resulting in fast polymer crystallization, giving origin to needle obstruction and non-continuous electrospun polymer processing. In that sense, DMF (with higher dielectric constant and lower volatility, see table 1) was added to the solution, allowing to solve the aforementioned problems and resulting in stable and continuous processing of the fiber membranes.

Table 1 - Physical and thermal properties of the solvents used for the processing of the fibers. Data collected from the material datasheet supplied by the manufacturers.

\begin{tabular}{cccccc}
\hline Solvent & $\begin{array}{c}\text { Melting } \\
\text { Point }\end{array}$ & $\begin{array}{c}\text { Boiling } \\
\text { point }\end{array}$ & $\begin{array}{c}\text { Dipole } \\
\text { moment }\end{array}$ & $\begin{array}{c}\text { Dielectric } \\
\text { constant }\end{array}$ & density \\
\hline & ${ }^{\circ} \mathrm{C}$ & ${ }^{\circ} \mathrm{C}$ & Debye & & $\mathrm{g} / \mathrm{cm}^{3}$ \\
DMF & -61 & 153 & 382 & 38.2 & 0.944 \\
CF & -63 & 62 & 1.15 & 4.8 & 1.483 \\
\hline
\end{tabular}

The influence of the applied electric field was investigated keeping constant the value of the inner needle diameter at $0.5 \mathrm{~mm}$ and a flow rate of $10 \mathrm{~mL} \cdot \mathrm{h}^{-1}$. The morphology of the obtained samples is represented in figure 1. A histogram (figure $1 \mathrm{c}$ and $\mathrm{d}$ ) of the 
fiber diameter distribution, determined from the SEM images and fiber average size and standard deviation was obtained. The as-spun membranes presented a fibrillar structure, without bead formation, with a smooth surface and with randomly oriented fibrils on the static grounded collector.
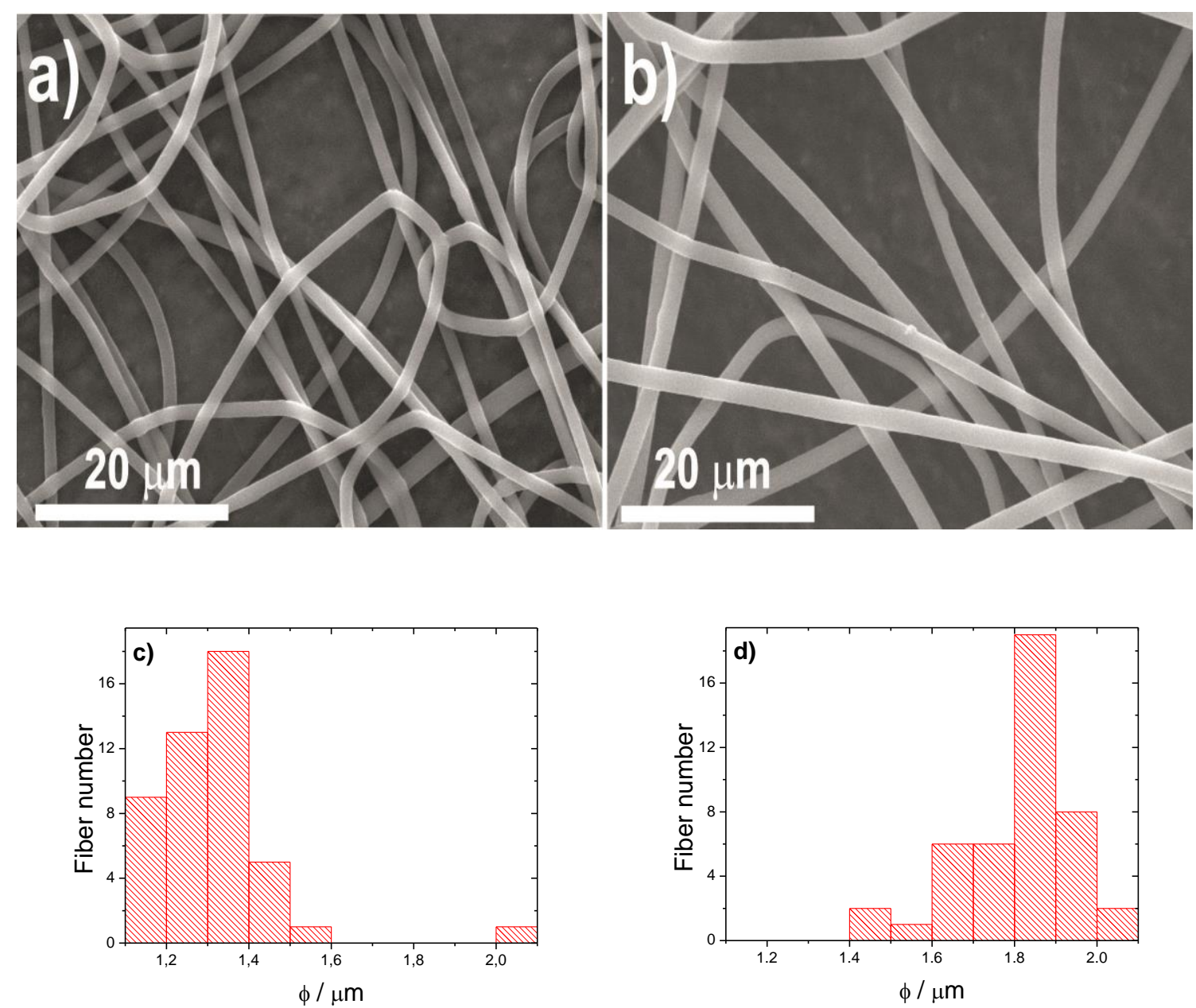

Figure 1 - PHB electrospun membranes obtained at 10/90 (10 \% PHB + 90\% solvent blend) with a needle inner diameter of $0.5 \mathrm{~mm}$ and a flow rate of $10 \mathrm{~mL} \cdot \mathrm{h}^{-1}$ at $1 \mathrm{kVcm} .^{-1}$ (a) and c)) and $1.75 \mathrm{kVcm} \cdot{ }^{-1}$ (b) and d)).

Samples obtained at several applied electric fields between the needle tip and the ground metallic collector showed a mean diameter that ranges between $1.31 \pm 0.13 \mu \mathrm{m}$ and $2.01 \pm 0.17 \mu \mathrm{m}$ (figure 2). An increase of the mean fiber diameter with increasing of the applied electric field was observed until $1.5 \mathrm{kV} . \mathrm{cm}^{-1}$. By further increasing the 
applied electric field, a decrease of the mean fiber diameter occurred (figure 2a) due to changes in mass flow and jet dynamics promoted by the applied electric field [15].

The formation of the thin fibers is mainly due to the stretching and acceleration of the jets promoted by the high electric field $[16,18]$ which in turn will result in higher charge density on the surface of the jets, increasing jet velocity and consequently fiber stretching. In this sense, it is generally reported that the diameter of the fibers becomes gradually smaller with increasing the applied electric field [19-22]. On the other hand, as observed in the present experiments and in other cases reported in the literature [15], this result is not general since increasing charge density within the applied electric field also affect other processing parameters such as jet traveling time, which has the opposite effect on the fiber diameter [23]. In order to promote the formation of independent and smooth fibers, most of the solvent evaporation must occur during the travel between the tip and the metallic ground collector. Increasing electric field decreases travelling time. Furthermore, increasing the applied electric field often enhances jet instability, promoting multiple fiber formation, which results in a broader distribution of the fiber diameters [16, 20,24].

\section{Figure 2 -}

The inner diameter of the needle also plays an important role in the electrospun fiber size and distribution. Typically, a small internal diameter reduces clogging and prevents the formation of beads on the electrospun fibers as well due to less exposure of the solution to the atmosphere during processing [25].

For PHB it was found that increasing needle inner diameter is associated to a decrease of the fiber mean diameter and size distribution becomes broader (figure 2b). Literature shows contradictory results concerning the influence of the internal needle diameter. Macossay et al. [15] found no influence of the needle diameter on average fiber diameter for poly(methyl methacrylate) electrospun fibers, but on the other hand, some authors report that a decrease of the needle inner diameter is associated to a reduction of the of fiber diameter, and such behavior was attributed to the increasing surface tension of the droplet, which results in variations of the forces needed for jet initialization and therefore in traveling time, influencing jet stretching and solvent 
evaporation. For PHB electrospun fibers, the increase of the needle inner diameter results in a formation of multiple jets that arise from the Taylor cone at the needle tip surface and consequently a reduction of the fiber average diameter and its broad distribution (figure $2 b$ ).

The influence of the solution feed rate on electrospun fiber mean diameter and distribution was also analyzed keeping constant the applied voltage $(25 \mathrm{kV})$ and the needle inner diameter $(0.5 \mathrm{~mm}$, figure 3$)$.

It is observed that increasing feed rate from 5 up to $10 \mathrm{~mL} \cdot \mathrm{h}^{-1}$ increases the mean fiber diameter, but by further increasing flow rates a slight decrease of the mean fiber diameter occurs (figure 3). On the other hand, fiber size distribution is very similar for the samples collected at different feed rates. It is generally expected increasing fiber diameters and number of beads when the feed rate increases due to the larger volume of solution that is drawn away from the needle. This increase of volume drawn from the needle tip will promote an increase of the time for the solvent to evaporate and consequently more time is needed for the polymer to crystallize, given origin to higher fiber diameters and broader distribution as observed for other polymer systems like PVDF [16], PLA [24] and chitosan [23].

For PHB, it seems that the low boiling point of the CF/DMF solvent blend (table 1) allows a fast evaporation during the flight time. In this situation, full solvent evaporation occurs when the fiber reaches the grounded collector and therefore the feed rate does not have strong influence on fiber diameter. It is also observed that for feed rates higher than $20 \mathrm{~mL} \cdot \mathrm{h}^{-1}$ the jet becomes instable and the fiber reaches the ground metallic collector with some solvent promoting the dissolution of the fibers already collected.

\section{Figure 3 -}

In order to study the influence of the polymer concentration of the CF/DMF solution, different polymer/solvent ratios were prepared. For an amount of $5 \%$ PHB on solvent dissolution, electrospinning was not feasible due to the high conductivity of the solution that promotes sparks. Increasing $\mathrm{PHB}$ concentration to $7 \%$ allows stable polymer electrospinning. Moreover, a decrease of the fiber diameter from $1.8 \pm 0.2 \mu \mathrm{m}$ (figure 1) 
down to $530 \pm 140 \mathrm{~nm}$ was observed and the fibers present same randomly distribution, with smooth fibers, without ribbons or beads (figure 4) observed for the polymer concentration of $10 \%$ (figure 1 )

\section{Figure 4 -}

\section{b) Stability against processing conditions}

Fourier transform infrared spectroscopy (FTIR) was used to monitor variations at a molecular level that might occur due to the electrospun conditions. Figure 5 shows the characteristic infrared spectra observed for PHB film and for the electrospun fibers obtained at different applied electrical fields. The overall response of the FTIR spectra is quite similar, no vibration modes are totally suppressed and no new modes seems to appear due to the changes in the processing parameters (figure 5), i.e. the structure and the crystalline phase of the PHB remain the same, independently of the processing conditions.

\section{Figure 5 -}

PHB absorption bands and their assignments are summarized in table 2. The results obtained showed that the most characteristic absorption peaks are the carbonyl and hydroxyl groups at $\sim 1720$ and $3278 \mathrm{~cm}^{-1}$, respectively. Other characteristic bands for PHB polymer appear at $\sim 1276, \sim 1180$, and $\sim 1057 \mathrm{~cm}^{-1}$, which can be assigned to the ester groups of the polymer (figure 5).

Table 2 - Characteristic FTIR absorption bands and assignments for PHB polymer [9].

Differential scanning calorimetry (DSC) normalized thermograms (heat flow divided by sample mass and heating rate) are shown in figure $5 \mathrm{~b}$. The first heating scan shows a 
strong endothermic peak around $170{ }^{\circ} \mathrm{C}$ that corresponds to melting. On the second scan, recorded after cooling from the melt at $10{ }^{\circ} \mathrm{C} / \mathrm{min}$, the glass transition appears around $0{ }^{\circ} \mathrm{C}$, and at higher temperatures, a cold crystallization exotherm process is observed in the range of $30 \pm 60{ }^{\circ} \mathrm{C}$, with a maximum at $45^{\circ} \mathrm{C}$. The sample after electrospinning was kept at room temperature for several weeks. Taking into account that the room temperature is higher than $\mathrm{T}_{\mathrm{g}}$, this annealing produces an increase of the crystalline phase of the PHB polymer in the as-spun membranes, this effect was observed by other authors in PHB films [26, 27]. Crystallinity degree of PHB electrospun mats can be calculated according to equation 1 :

$$
\Delta X_{c}=\frac{\Delta H_{\text {sample }}}{\Delta H_{0}^{f}} * 100
$$

where, $\Delta H_{\text {sample }}$ and $\Delta H_{0}^{f}(=146 \mathrm{~J} / \mathrm{g}[26,27])$ are the experimental melting enthalpy and the thermodynamic melting enthalpy of a $100 \%$ crystalline PHB polymer, respectively. Both PHB samples, film and electrospun membrane show the same crystallinity degree of $53 \%$, which is similar to the values reported in literature. Xu et al. [27] performed PHB annealing measurements at different temperatures above glass transition and found a maximum crystallinity degree of $56.8 \%$. Amico et al. [28] study the thermal properties of the PHB filled with clays and reported a crystallinity of $66 \%$ for pure polymer that slightly decreases with the incorporation of the clays into the polymer matrix.

Thermal instability is one of the major drawbacks of the PHB since the decomposition temperature of the polymer is close to the melting point. Figure 6 shows the TGA data obtained for the PHB fiber mats at various heating rates. A major weight loss process was observed between 200 and $350{ }^{\circ} \mathrm{C}$. No loss of volatiles was found below $200{ }^{\circ} \mathrm{C}$ showing the absence of solvents remains used in electrospinning process and water (due to the hydrophobic nature of the polymer). Two main temperatures were revealed: the initial degradation temperature $\left(\mathrm{T}_{\text {initial }}\right)$, which is defined as the temperature at which the experimental curves deviates from the tangent line that the mass evolution follows before degradation, and the onset temperature ( $\left.\mathrm{T}_{\text {onset }}\right)$ that is calculated by extending the pre-degradation portion of the curve to the point of the interception with a line drawn as a tangent to the steepest portion of the mass curve occurring during degradation. 
Figure 6 -

The kinetics of the mass loss process can be investigated by analyzing experiments performed at different heating rates (figure $6 a$ and $b$ ). As expected, increasing heating rate shifts the onset temperature of the degradation process to higher temperatures, not affecting any of the main characteristics of the process itself. A typical model for the decomposition has the following general form:

$$
\frac{\partial \alpha(t)}{\partial t}=k(T) f[\alpha(t)]
$$

where, $\alpha$ represents the degree of conversion of the sample under degradation, defined by:

$$
\alpha=\left(w_{0}-w_{t}\right) /\left(w_{0}-w_{\infty}\right)
$$

where $w_{0}, w_{(t)}$ and $w_{\infty}$ are the weights of the sample before degradation, at a given time $t$ and after complete degradation, respectively. The rate constant $k(t)$ changes with the absolute temperature according to the Arrhenius equation. $f(\alpha)$ represents the net results of elementary steps, as the polymer degradation is often a chain reaction. For the reaction model $f(\alpha)=(1-\alpha)^{n}$, where $n$ is the reaction order, assumed to remain constant during the degradation process.

The isoconversional method of Ozawa-Flynn-Wall (OFW) $[29,30]$ is a method which assumes that the conversion function $f(\alpha)$ does not change with the variation of the heating rate for all values of the degree of conversion $\alpha$. It involves measuring the temperatures corresponding to fixed values of $\alpha$ from the experiments at different heating rates, $\beta$. In this theory:

$$
\ln (\beta)=\frac{\ln \left(A E_{\text {act }}\right)}{R}-\ln [f(\alpha)]-\frac{E_{\text {act }}}{R T}
$$


where $A$ is a pré-exponential factor $\left(\mathrm{min}^{-1}\right), \mathrm{R}$ is the gas constant $\left(8.31 \mathrm{~J} \cdot \mathrm{mol}^{-1} \cdot \mathrm{K}^{-1}\right)$, and $E_{\text {act }}$ is the activation energy of the degradation process. By the plotting $\ln (\beta)$ vs $1 / T_{T}$, activation energy can be obtain from the slope of the resulting straight line, regardless of the reaction order of the system. The validity of this model is based in the assumption that the conversion at the peak maximum is constant for the different heating rates [29, 30]. The linear fitting for the PHB as-spun mats is represented in figure 6c. The evolution of the activation energy with the degree of conversion reveals to be constant and a value of $91 \mathrm{~kJ} / \mathrm{mol}$ was obtained. Previous reports have shown that the activation energy value of the degradation fall within a wide range $100-235 \mathrm{~kJ} / \mathrm{mol}$. A clear explanation for the wide variation of $E_{\text {act }}$ has yet to be provided. Despite this uncertainty, the random $\beta$-elimination scission has been widely held as the exclusive degradation mechanism of PHB based on typical structures of pyrolysis products, i.e. crotonic acid and oligomers with a crotonate end-group [31, 32]. Recently, an E1cB mechanism proceeding via $\alpha$-deprotonation by a carboxylate anion to produce the same products was proposed [33].

\section{c) Cell viability}

Fiber mats surface energy was estimated by water contact angle measurements on a PHB film and in the as-spun membranes. The water contact angle measured for the polymeric film was $77 \pm 2^{\circ}$, similar to the values reported in the literature [34]. For the as-spun membranes the calculated contact angle was $126 \pm 3^{\circ}$, which reveals a strong increase of the hydrophobicity due to morphological variations. This behavior is common to other polymeric systems obtained by electrospinning such as PLA [35].

PHB shows potential for biomedical and tissue engineering applications such as bone repair and drug-carriers [36]. Electrospinning uses many times toxic solvents that could eventually prevents the use of electrospun membranes in biomedical applications. MTT tests were used to characterize proliferation and viability of cells on PHB as-spun fiber membranes throughout 3 days of culture. The absorbance (A) was measured at $570 \mathrm{~nm}$ for all the samples at each time. It was observed for the first 3 days that electrospun PHB fiber mats prepared under the above described method and conditions do not inhibit the adhesion of cells (figure 7). 


\section{Figure 7 -}

\section{Conclusions}

PHB was dissolved in a mixture of chloroform (CF) and dimethylformamide (DMF) and processed by electrospinning at $40{ }^{\circ} \mathrm{C}$. The addition of DMF to the solvent solution turns the polymer membrane more stable. Smooth randomly oriented fibers without beads and ribbons were obtained. Moreover, it was observed that an increase of the applied electric field is associated to an increase of the fiber average diameter until a mean diameter of $2.01 \mu \mathrm{m}$ for an applied electric field of $1.50 \mathrm{kV} . \mathrm{cm}^{-1}$, but for higher electric fields, a decrease of the fiber diameter was observed, due to the jet instability and fiber stretching. On the other hand, the increase of the tip inner diameter promotes a decrease of the fiber average diameter and a broader distribution. Fiber around $500 \mathrm{~nm}$ was obtained by decreasing down to $7 \%$ the concentration of PHB in the solution.

Infrared spectroscopy showed that the changes in the processing parameters do not alter the crystalline phase present in the polymer. Moreover, the crystallinity of PHB film and as-spun membranes are similar, around $53 \%$. Thermogravimetric results of the asspun membranes show that polymer degradation occurs in single step degradation process, with activation energy of $91 \mathrm{~kJ} / \mathrm{mol}$.

MC-3T3-E1cell adhesion was not inhibited by the fiber mats preparation, indicating the suitability of the material for biomedical applications.

\section{Acknowledgements}

This work is funded by FEDER funds through the "Programa Operacional Factores de Competitividade - COMPETE" and by national funds arranged by FCT- Fundação para a Ciência e a Tecnologia, project references NANO/NMed-SD/0156/2007, PTDC/CTM/73030/2006 and PTDC/CTM/69316/2006. The authors also thank support from the COST Action MP1003, 2010 'European Scientific Network for Artificial Muscles'. V.S. and D.M.C thanks the FCT for the SFRH/BPD/63148/2009, SFRH/BD/ $82411 / 2011$ grants respectively. C. R. thanks the IINL for a financial support via a $\mathrm{PhD}$. 
JLGR acknowledge the support of the Spanish Ministry of Science and Innovation through project No. MAT2010-21611-C03-01 (including the FEDER financial support). CIBER-BBN is an initiative funded by the VI National R\&D\&i Plan 2008-2011, Iniciativa Ingenio 2010, Consolider Program, CIBER Actions and financed by the Instituto de Salud Carlos III with assistance from the European Regional Development Fund.

\section{References}

[1] R Langer, J Vacanti (1993) Science 260: 920. Doi:10.1126/science.8493529

[2] MT Khorasani, SA Mirmohammadi, S Irani (2011) International Journal of Polymeric Materials 60: 562. Doi:Pii 938486575

10.1080/00914037.2010.531809

[3] K Sombatmankhong, N Sanchavanakit, P Pavasant, P Supaphol (2007) Polymer 48: 1419. Doi:10.1016/j.polymer.2007.01.014

[4] M Wróbel, J Zebrowski, J Szopa (2004) Journal of Biotechnology 107: 41. Doi:10.1016/j.jbiotec.2003.10.005

[5] AQ Ali, TP Kannan, A Ahmad, AR Samsudin (2008) Toxicology in Vitro 22: 57. Doi:10.1016/j.tiv.2007.08.001

[6] MK Gouda, AE Swellam, SH Omar (2001) Microbiological Research 156: 201. Doi:10.1078/0944-5013-00104

[7] KC Reis, J Pereira, AC Smith, CWP Carvalho, N Wellner, I Yakimets (2008) Journal of Food Engineering 89: 361. Doi:10.1016/j.jfoodeng.2008.04.022

[8] M Dias, MC Moraes Antunes, AR Santos, Jr., MI Felisberti (2008) Journal of Materials Science-Materials in Medicine 19: 3535. Doi:10.1007/s10856-008-3531-1

[9] AS Asran, K Razghandi, N Aggarwal, GH Michler, T Groth (2010) Biomacromolecules 11: 3413. Doi:10.1021/bm100912v

[10] Y-W Wang, Q Wu, J Chen, G-Q Chen (2005) Biomaterials 26: 899. Doi:10.1016/j.biomaterials.2004.03.035

[11] L Nivison-Smith, J Rnjak, AS Weiss (2010) Acta Biomaterialia 6: 354. Doi:10.1016/j.actbio.2009.08.011

[12] K Sombatmankhong, O Suwantong, S Waleetorncheepsawat, P Supaphol (2006) Journal of Polymer Science Part B-Polymer Physics 44: 2923. Doi:10.1002/polb.20915

[13] P Sangsanoh, S Waleetorncheepsawat, O Suwantong, et al. (2007) Biomacromolecules 8: 1587. Doi:10.1021/bm061152a

[14] R Fryczkowski, T Kowalczyk (2009) Synthetic Metals 159: 2266. Doi:10.1016/j.synthmet.2009.09.008

[15] S. Ramakrishna, K. Fujihara, W.-E. Teo, T.-C. Lim, Z Ma(2005). (2005) An introduction to electrospinning and nanofibers. World Scientific, New Jersey

[16] C Ribeiro, V Sencadas, JL Gomez Ribelles, S Lanceros-Mendez (2010) Soft Materials 8: 274. Doi:10.1080/1539445x.2010.495630

[17] C Wang, Y-W Cheng, C-H Hsu, H-S Chien, S-Y Tsou (2011) J Polym Res 18: 111. Doi:10.1007/s10965-010-9397-1

[18] X-H Qin, Y-Q Wan, J-H He, J Zhang, J-Y Yu, S-Y Wang (2004) Polymer 45: 6409. Doi:10.1016/j.polymer.2004.06.031 
[19] S Zhao, X Wu, L Wang, Y Huang (2004) Journal of Applied Polymer Science 91: 242. Doi:10.1002/app.13196

[20] K Gao, X Hu, C Dai, T Yi (2006) Materials Science and Engineering: B 131: 100. Doi:10.1016/j.mseb.2006.03.035

[21] MM Demir, I Yilgor, E Yilgor, B Erman (2002) Polymer 43: 3303. Doi:10.1016/s00323861(02)00136-2

[22] S Megelski, JS Stephens, DB Chase, JF Rabolt (2002) Macromolecules 35: 8456. Doi:10.1021/ma020444a

[23] V Sencadas, DM Correia, A Areias, et al. (2012) Carbohydrate Polymers 87: 1295. Doi:10.1016/j.carbpol.2011.09.017

[24] C Ribeiro, V Sencadas, C Miguel Costa, JL Gomez Ribelles, S Lanceros-Mendez (2011) Science and Technology of Advanced Materials 12. Doi:10.1088/1468-6996/12/1/015001

[25] XM Mo, CY Xu, M Kotaki, S Ramakrishna (2004) Biomaterials 25: 1883. Doi:10.1016/j.biomaterials.2003.08.042

[26] J Kemnitzer, R Gross, S McCarthy, J Liggat, D Blundell, M Cox (1995) Journal of Polymers and the Environment 3: 37. Doi:10.1007/bf02067792

[27] S Xu, R Luo, L Wu, K Xu, G-Q Chen (2006) Journal of Applied Polymer Science 102: 3782. Doi:10.1002/app.24742

[28] DA D'Amico, LB Manfredi, VP Cyras (2012) Journal of Applied Polymer Science 123: 200. Doi:10.1002/app.34457

[29] JH Flynn, LA Wall (1966) Journal of Polymer Science Part B: Polymer Letters 4: 323.

[30] T Ozawa (1965) Bulletin of the Chemical Society of Japan 38: 1881.

[31] H Morikawa, RH Marchessault (1981) Canadian Journal of Chemistry 59: 2306. Doi:10.1139/v81-334

[32] A Ballistreri, D Garozzo, M Giuffrida, G Impallomeni, G Montaudo (1989) Journal of Analytical and Applied Pyrolysis 16: 239. Doi:10.1016/0165-2370(89)80028-2

[33] M Kawalec, G Adamus, P Kurcok, et al. (2007) Biomacromolecules 8: 1053. Doi:10.1021/bm061155n

[34] DM Zhang, FZ Cui, ZS Luo, YB Lin, K Zhao, GQ Chen (2000) Surface and Coatings Technology 131: 350. Doi:10.1016/s0257-8972(00)00810-0

[35] AC Areias, C Ribeiro, V Sencadas, et al. (2012) Soft Matter.

[36] G-Q Chen, Q Wu (2005) Biomaterials 26: 6565. Doi:10.1016/j.biomaterials.2005.04.036 